\title{
Teaching Application Area Oriented Mathematics in Engineering
}

\author{
Andreas Körner ${ }^{1}$, Stefanie Winkler ${ }^{1}$ \\ ${ }^{1}$ Institute of Analysis and Scientific Computing, TU Wien, Wiedner Haupstraße 8-10, 1040 Vienna, Austria; \\ *andreas.koerner@tuwien.ac.at
}

\begin{abstract}
The article focuses on the symbiosis between mathematics in engineering education and problem based approaches. Traditional approaches focus on the idea to teach first basic math and establish on this basics the theoretical subjects out of the application area, e.g. theoretical foundation, theoretical physics, mechanics, etc.

The obvious benefit is the formal foundation and the structured development of the theory. The disadvantage is a delay in developing the core subject after the mathematical and physical foundations are grounded.

In the subject of technical physics at TU Wien the approach is different. The first semester consists of three different mathematics subjects, analysis, linear algebra and a subject called practical mathematics. The practical math is supplying the fourth subject with the necessary tools for understanding the basic concepts of physics.

This paper is introducing this concept in detail and analyzing the possibilities to apply this approach in engineering mathematics. The last section of the paper addresses the relation to modelling and simulation as a subject in STEM studies and points out the parallel concepts and their application.
\end{abstract}

\section{Introduction}

The basic concept in including the topics and methods of mathematics in one engineering subject is introduced at the HAW Hamburg since 2014, see [1]. The core idea is to provide mathematical topics and apply them the next week in one of the subjects of engineering. This article goes one step further, that mathematics is split in theory and application and the applied math course builds the foundation for engineering subject.
A new approach would be to combine applied mathematics and a basic subject from the engineering field in the same semester, almost parallel. Math content, which has been taught 1 or 2 weeks ago, would be applied in the engineering subject immediately after. The benefit is that students see the application just in time.

For lecturers a benefit is that the examination during the semester can be less, because the corresponding subject is using the methods and students need to know them to pass this subject.

A particular example to illustrate the basic idea is the subject physics foundation. Almost all engineering studies have a basic physics education in the first semester. These physics courses usually are taught on a high school level, because the necessary higher mathematics for teaching e.g. mechanics, are missing.

The splitting into applied and theoretical mathematics would result in the effect that the methods, which are required in the physics course and the structural theoretical mathematics, shall be given in separate courses. Without studying the one subject it will not be possible to understand the other subject, see [2] as well. This interconnection will substitute the motivation aspects of the mathematics. Hence, students need to study methods and algorithms for the applied engineering courses.

This setup decreases the overall efforts for students, if the mathematics courses and the applied courses are coordinated regarding their requirements. Furthermore, the connection between mathematics and the applied courses can be supported by online examples in exercises in the math course and in the best case in the applied courses as well. 


\section{Physics Studies in contrast to Engineering Studies}

First, it should be argumented, why physics is more accessible for a different curriculum approach. This can be shown by analyzing the content of the basic physics course.

The covered physics topics are:

1. Mechanics of a particle

2. Collisions of particles

3. Rigid body dynamics

4. Kinematic reference systems

5. Oscillations and waves

These headlines cover several mathematical aspects, like the concept of forces and potentials in (1) and (2). Students have to deal with line integrals and vector fields after few weeks of the start of the first semester. For chapter (3) higher dimensional integration techniques are required and for (5) even ordinary and partial differential equations.

To compare to an engineering subject, e.g. electrical engineering, the basic subject of electrodynamics is given in the fourth semester of bachelor study. The basic math courses are accommodated in the first three semesters, so in the fourth semester allow to apply the whole math package.

Disadvantage in the engineering education is the lack of application. The three sequential math courses are preparing the whole bunch of methods on a stock. Nevertheless, the mathematical requirements are comparable to the ones in physics.

Covered topics in electrodynamic are:

1. Electromagnetic fields

2. Energy and impulse

3. Stationary and quasi stationary fields

4. Magnetic induction

5. Electromagnetic waves

The requirements from the mathematical point of view is comparable. For (3) and (4) vector calculus and vector analysis is needed. The difference is, that the physics subject is offered in the first semester, meanwhile the electrodynamic subject is offered in the fourth.

\section{Setup Requirements and Curriculum}

For applying this approach of collaborative teaching, some requirements are necessary:

- Flexibility in Curriculum

- Collaborative Teacher in the first semesters

- Itemized requirements for related subjects

- Perfect synchronized lectures and exercises

- Harmonized content

\subsection{Traditional Curricilum Setup}

Most Curriculums are going the traditional way and form, first the mathematical basics (and basic in physics) and continue, based on these basics, to construct the applied engineering theory.

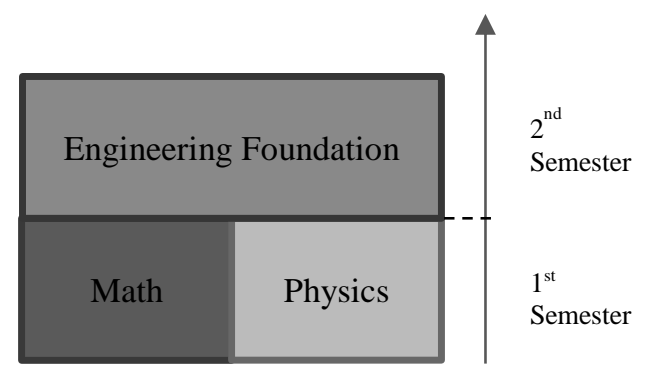

Figure 1: Each Box is illustrating a subject, e.g. Math and Physics, as a basis for a particular Engineering Subject, e.g. electrodynamics or analytic mechanics.

The strategy of building up, for an engineering subject, is illustrated in Figure 1. Mechanical and electrical engineering studies are typically structured in this traditional approach.

\subsection{Integrated Curriculum Setup}

Physics studies are different in this aspect. The basics in physics are concerning much more fields like mechanics, thermodynamics, optics, electrodynamics, relativity, etc. than the typical engineering studies. To wait for the math subjects to build the basics would last too long, so the math is split in two complementary subjects.

The theoretical math subjects, like analysis and linear algebra, on the one side and practical mathematics on the other.

The last subject equips the students with methods and practices to compute different mathematical problems and the theoretical subjects are going in the conceptual deepness of the content. 
The resulting curriculum needs to have small granularity, to equip the physics course with the mathematical methods needed. Figure 2 is illustrating this structure of the curriculum.

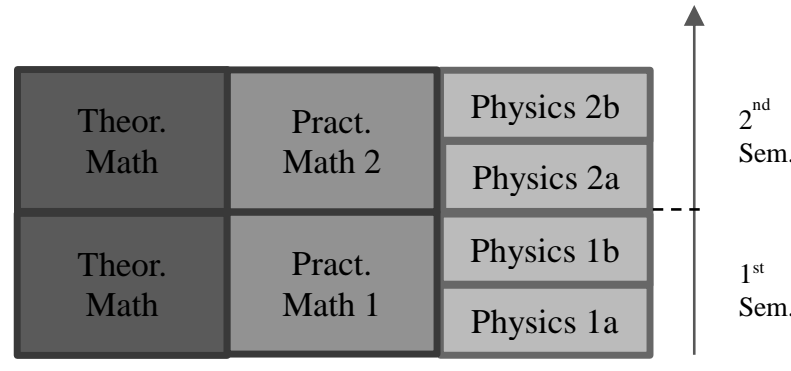

Figure 2: Each Box is illustrating a subject, e.g. Math and Physics, as a basis for a particular Engineering Subject, e.g. electrodynamics or analytic mechanics.

Of course, the curriculum of physics studies is different to the classical engineering studies, but the approach to split the calculus skills, from the theoretical math knowledge provides faster math concepts for engineering application.

Moreover, the mentioned benefits, the presented way of interconnecting mathematical skills and physics is benefiting the whole physics education, as presented in [4]. The same aspects are addressed in the highschool level in [5].

\subsection{A demonstrative Example}

A particular example in electrical engineering is the electric flux. To understand the relation between flux $\Psi$ and flux density $\vec{D}$, which is the representing quantity, who is measured in practical examples, one needs vector calculus. In particular, the relation is given by a surface integral

$$
\Psi=\int_{\mathcal{A}} \vec{D} \cdot d A
$$

The development of this mathematical concept is a long one and needs in the classical setup 3 semesters. If there is a practical mathematics, which is motivating the surface integral by geometrical facts and reduces them to the computation of particular examples, the development of such a method could work faster.

Especially in engineering subjects, mathematical concepts are often reduced by using symmetries, simplifications and specialisations; there is never a situation to apply an abstract theory.

\section{Learning Perspective versus Academic Buildup}

Discussing these issues about the setup of the curriculum raises the argument of academic buildup. Of course, the curricula of higher education institutions should have a certain buildup, but the proposed setup would split this buildup just in two tracks: Skills and deductive math. The skills equip the students earlier in to be able to understand engineering applications and the deductive math path is illustrating the power of an abstract approach and illustrates the benefit of such access. Additionally, the previous learned skills can make the step to the abstract deductive approach much easier.

Apart from the academic discussion, several applied fields of STEM studies are applying a similar concept. One famous field is robotics. Robotics is starting in a simple setup, understandable in a geometrically way by almost all people of the society. More in detail, the robotics field is much more physics and math related, than it looks like in the beginning. In [3] is presented the idea of practical robot education. This approach is following some ideas presented in this paper, which is well accepted in the scientific setting.

\section{Modelling and Simulation}

A similar setup is given in the interdisciplinary subject of modelling and simulation. That subject is in the methodological layer influenced by mathematic knowledge and in the application layer by physics and engineering.

The related teaching approach is closely related to the situation faced in a modelling and simulation lecture. Students need to apply the math basics in a particular application field. Even in this setup computer simulations are used to help students get familiar with the application field and gather experiences in modelling. 


\subsection{Matheamtical Modelling}

Addressing mathematical modelling is a similar scenario to the basic subject physics and engineering. Students need to study basic analysis and calculus, advanced linear algebra, numeric and computer numeric, differential equations, dynamic systems and partial differential equations before starting with modelling and simulation subjects. Normally this requires at least 5 semester of bachelor studies in mathematics. To attract students earlier to modelling and simulation, in the traditional way, is problematic. One approach to succeed in it would be the earlier introduction of calculus and physical modelling.

\subsection{Numerical Simulation}

Numerical Simulation requires more than solely mathematical skills. In several simulation environments are required programming skills.

A simple use case is the implementation of a particular mathematical model in MATLAB. Numerical mathematics meets programming on a higher level to implement a model, which is given by a mathematical environment, e.g. differential equation, differential-algebraic equations, automata, etc.

A problem-based teaching approach in this field can lead to developing the necessary mathematical concepts for the specific example. Developing the corresponding skills in solving math and implementing the related simulation model would generate a completed knowledge in this area.

\section{Conclusion and Outlook}

The paper presented a new approach on how to design the math education in engineering subjects. The motivation of this approach is the curriculum of technical physics at TU Wien.

The proposed structure of a linked course system would benefit from the progress in the linked subjects and is not relying on the topics presented in the own course. There are several advantages and disadvantages.

One advantage is obvious, the evolution in methods and techniques in the math courses, that can be applied in the technical course immediately. Students could have more insight, why to study math and what will they benefit from a stable math education in the field of STEM subjects.

One major disadvantage is, that if there is any serious problem in the math course to understand particular methods or techniques, students may have also troubles in the applied subject. Being that math is considered as one of the difficult subjects, it should be investigated in a survey over one semester.

Other problems can cause the requirement of timed and synchronized course progress. If one subject is delayed, due to holidays or canceled lectures, the other subjects are suffering from this.

In the last section, the concept was extended to the subject of modelling and simulation. Several similar requirements can be applied in this field of interest and the ideas of teaching an integrated subject of a smaller field, can lead to an earlier access to the field of (mathematical) modelling and simulation.

Further work will concern some survey on the success of the students in technical physics at TU Wien. Moreover, in a subject of mathematical modelling in system simulation, the problem based approach will be applied to the exercises part of the course.

\section{References}

[1] Ditzel B, Dahlkemper J, Landenfeld K, Renz W. Integratives Grundstudium in den Ingenieurwissenschaften durch Themenwochen - vom Konzept zur Umsetzung. ZfHE 9-4, 2014

[2] A. Renkl, R. K. Atkinson, U. H. Maier, und R. Staley, „From Example Study to Problem Solving: Smooth Transitions Help Learning“, The Journal of Experimental Education, Bd. 70, Nr. 4, S. 293-315, 2002.

[3] K. Nagai, "Learning while doing: practical robotics education," in IEEE Robotics \& Automation Magazine, vol. 8, no. 2, pp. 39-43, June 2001, doi: 10.1109/100.932756.

[4] Uhden, O., Karam, R., Pietrocola, M. et al. Modelling Mathematical Reasoning in Physics Education. Sci \& Educ 21, 485-506 (2012). https://doi.org/10.1007/s11191011-9396-6

[5] Teodoro, V., Neves, R. Mathematical modelling in science and mathematics education in Computer Physics Communications, Volume 182, Issue 1, 2011, p. 8-10, https://doi.org/10.1016/j.cpc.2010.05.021. 